\title{
MINIMIZAÇÃO DOS CUSTOS DE FRETE NA DISTRIBUIÇÃO DE CIMENTO POR PROGRAMAÇÃO LINEAR
}

\author{
MINIMIZING FREIGHT COSTS IN THE CLEANING DISTRIBUTION \\ BY LINEAR PROGRAMMING
}

\section{Recebimento: 15/07/2017- Aceite: 10/11/2017- Publicação: 28/01/2018 \\ Processo de Avaliação: Double Blind Review}

Igor Pereira da Silva ${ }^{1}$

Graduado em Logística

Faculdade de Tecnologia de Guarulhos

igor719@gmail.com

Nemesio Rodrigues Capocci

Graduado em Logística

Faculdade de Tecnologia de Guarulhos

nemesio.capocci@fatec.sp.gov.br

Valdir Cardoso Gonçalves

Graduado em Logística

Faculdade de Tecnologia de Guarulhos

valdir_goncalves87@yahoo.com.br

Marcos José Corrêa Bueno

Mestrado em Engenharia de Produção

Faculdade de Tecnologia de Guarulhos

marcosjcbueno@gmail.com

Marcos Antonio Maia de Oliveira

Doutorado em Administração

Faculdade de Tecnologia de Guarulhos

marcos.maia@fatec.sp.gov.br

\section{RESUMO}

O presente estudo teve como objetivo demonstrar a redução dos custos de distribuição de cimentos por meio de programação linear. Trata-se de um estudo de caso sobre a aplicação de métodos quantitativos com Diagrama de Rede para determinar o roteiro ótimo a ser realizado, que corresponda ao menor custo de frete possível para distribuição de sacos de cimento de $50 \mathrm{~kg}$ de 2 CD's - centros de distribuições para 6

\footnotetext{
${ }^{1}$ Autor para correspondência: Faculdade de Tecnologia de Guarulhos. R. João Gonçalves, 511 Centro, Guarulhos - SP, 07010-010. Brasil.
}

Revista ENIAC Pesquisa, Guarulhos (SP), V.7, n.1, jan.- jun. 2018. 
lojas da região metropolitana de São Paulo de uma empresa varejista de materiais para construção. Para efeito de comparação utilizou-se também o método de aproximação de Vogel para identificar qual o método mais adequado para este tipo de situação. Os resultados demonstraram otimização da distribuição de cimento em função dos custos. A relevância dessa análise está na possível utilização por empresas que enfrentem esta mesma situação na distribuição de rede.

Palavras-chave: Minimização; Distribuição; Programação; Linear.

\begin{abstract}
The present study aimed to demonstrate the reduction of cement distribution costs through linear programming. This is a case study on the application of quantitative methods with Network Diagram to determine the optimal route to be performed, corresponding to the lowest freight cost possible for the distribution of cement bags of $50 \mathrm{~kg}$ of 2 CD's - distributions to 6 stores in the metropolitan region of São Paulo from a retailer of construction materials. For comparison purposes, the Vogel approximation method was used to identify the most appropriate method for this type of situation. The results showed optimization of the cement distribution in terms of costs. The relevance of this analysis is the possible use by companies that face this same situation in network distribution.
\end{abstract}

Keywords: Minimization; Distribution; Programming; Linear.

\title{
1. INTRODUÇÃ̃
}

O âmbito empresarial necessita de novas competências e não basta uma resposta correta para uma pergunta complicada, pois, para um mesmo problema podem existir várias soluções possíveis e cada uma deve ser fornecida com informações consistentes e analisadas a fim de determinar, por meio da análise de cada solução possível a solução ótima visando minimizar o custo para resolução do problema. Em geral para uma empresa privada os custos logísticos situam-se em 19 e $22 \%$ das vendas liquidas e os custos de frete consome de $1 / 3$ à $2 / 3$ dos custos logísticos totais. Assim, a otimização dos resultados constitui um fator de extrema importância, principalmente se considerando o alto grau de competitividade com exigência do mercado atual.

O presente estudo de caso tem como demonstra por meio do uso da programação linear utilizada como ferramenta para apoio na tomada de decisão, visando a otimização do resultado com o menor custo de frete possível para a distribuição de sacos de cimento de $50 \mathrm{~kg}$ de uma empresa varejista de materiais para construção na região

Revista ENIAC Pesquisa, Guarulhos (SP), V.7, n.1, jan.- jun. 2018. 
metropolitana de São Paulo. Será utilizado o SOLVER que é uma ferramenta do Excel, para apresentação do resultado ao problema proposto. Após o resultado obtido através do SOLVER, será aplicado também o método de aproximação de Vogel para efeito de comparação e para determinar qual dos métodos aplicados atende melhor o problema explanado.

\section{LOGÍSTICA DE DISTRIBUIÇÃO E PROGRAMAÇÃO LINEAR}

Para Pedroso Neto et al, (2015), nas últimas décadas a economia dos países desenvolvidos transformou-se de forma acelerada com o intuito de atender as demandas dos clientes com o menor custo e tempo possíveis em razão da globalização e concorrência acirrada. Nesse cenário, para uma organização manter-se ativa e competitiva, se faz necessário reduzir ao máximo os custos e oferecer um bom nível de serviço aos seus clientes. Essa meta pode ser alcançada qualificando colaboradores, além de análise constante dos custos operacionais.

Conforme Pompermayer e Lima (2002), perante essa realidade, ainda muitas são as empresas tanto pequenas, médias e até grandes, que carecem de técnicas e ferramentas de tomadas de decisão para o auxílio na gestão dos negócios. Uma maneira eficaz para uma boa administração dos custos é a transmutação comportamental das organizações com base em técnicas modernas de gestão dos recursos produtivos, humanos e financeiros, pois sob quaisquer circunstâncias existe a possibilidade de redução dos custos. Vários são os setores com os quais o administrador deve se preocupar dentro de uma organização, não somente com a eficiência produtivo dos recursos envolvidos, mas também com os custos gerados.

Segundo Mckee (2013, p. 08) "O transporte representa um significativo custo logístico por parte das empresas absorvendo de um a dois terços do custo total”. No ponto de vista de Novaes (2007), a distribuição tem como objetivo levar os produtos certos para os locais corretos no tempo certo com o menor custo possível concomitantemente a um bom nível de serviço, contudo, existe um antagonismo entre redução de custo e melhoria do nível de serviço haja vista que melhorias em um determinado sistema comumente vêm acompanhadas de custos maiores seja na atividade de transporte, armazenagem ou mesmo no estoque.

Revista ENIAC Pesquisa, Guarulhos (SP), V.7, n.1, jan.- jun. 2018. 
Os estudos nas áreas de transportes têm relevância na atual realidade da globalização. A logística, na qual o transporte é normalmente seu principal componente, é vista como a última fronteira para a redução dos custos das empresas, enquanto, por outro lado, não se concebe uma política de desenvolvimento regional e nacional com a adequação da infraestrutura de transportes. (Caixeta-Filho, 2015, p. 88).

Conforme Fleury (2002), a atividade de transporte está intrinsecamente ligada à distribuição. A relevância dessa atividade pode ser mensurada com o auxílio de três indicadores financeiros, são eles faturamento, custo e lucro. A administração dessa atividade deve ser ampla cobrindo uma vasta gama de aspectos que se dividem em dois grupos, nível estratégico e nível operacional.

Martín (2003) expõe que surgiu com a segunda guerra mundial na Grã-Bretanha, onde administradores militares chamaram um grupo de cientistas de diversas áreas do conhecimento para estudarem os problemas táticos e estratégicos associados a defesa do país.

Ainda segundo Martín (2003), com os bons resultados obtidos pela GrãBretanha com estes estudos, militares norte-americanos levaram estes conceitos para os Estados Unidos. Após a guerra, administradores industriais começaram a aplicar as ferramentas de pesquisa operacional na resolução de problemas Industriais.

Segundo Marins (2011), essa ciência possui uma ampla utilização nas indústrias, governo, empresas de serviços e comerciais, tendo um enfoque sistêmico nos problemas gerenciais buscando a melhor solução possível, utilizando uma metodologia de trabalho em equipe que envolve matemática, estatística, engenharia, economia e computação.

Moreira (2010), afirma que a pesquisa operacional dispõe de muitas técnicas, entre elas está a programação linear ou otimização linear que é utilizado em diversos tipos de problemas como maximização de lucros, minimização de custos ou mesmo aplicações em rede tais como escala de produção, fluxo máximo, minimização de custos em transporte e distribuição, menor caminho dentre outros. Rezende Filho (2006) salienta que os avanços da pesquisa operacional nas últimas décadas se devem ao fato do desenvolvimento da computação que possibilitou a criação de softwares que por sua vez auxiliam de forma considerável os cálculos das diversas técnicas que essa ciência oferece, devido a sua alta capacidade de processamento e armazenagem dos dados.

Redes são diagramas compostos por uma coleção de vértices ou nós ligados entre si por um conjunto de arcos. Os nós são simbolizados por círculos e

Revista ENIAC Pesquisa, Guarulhos (SP), V.7, n.1, jan.- jun. 2018. 
representam os pontos de junção que conectam os arcos. Os arcos são representados por setas, que conectam os nós e revelam a direção do fluxo de um ponto a outro (LACHTERMACHER, 2009, p. 118).

Ainda segundo Lachtermacher (2009, p. 118), um problema de transporte é um tipo muito especial e comum de aplicação de programação linear e este, será utilizado para este estudo de caso.

Outra técnica utilizada para resolução deste tipo de problema é conhecida como método de Aproximação de Vogel (VAM - Vogel Approximation Method), que através de um algoritmo de cálculos consegue atingir uma solução próxima da solução ideal. Essa técnica consiste em selecionar células em uma matriz do transporte analisando o aumento da diferença entre os dois menores pontos horizontais e verticais na qual a célula selecionada é aquela que gerar a maior diferença em comparação com a célula de menor valor da vertical ou da horizontal.

\section{METODOLOGIA}

Foi utilizado o método de estudo de caso, essa modalidade de pesquisa de acordo com Gil (2006), consiste em um estudo profundo de um ou poucos objetos, de forma que possibilite um amplo conhecimento. Ocorre, porém, que esse método não fornece uma base sólida para generalização, contudo, o objetivo dessa modalidade de pesquisa é possibilitar uma visão global de um problema e não um conhecimento preciso das características de uma população.

Para este estudo de caso foi levantado um problema de abastecimento de 6 lojas de uma rede de varejo de materiais para construção e que partirá de 2 Centro logísticos: um localizado na cidade de Guarulhos na Rodovia Presidente Dutra, 224,8 km e outro na cidade de Santo André na Avenida Pereira Barreto. As lojas que receberão o produto que irá compor o seu estoque para atendimento dos clientes estão espalhadas pela cidade de São Paulo, Santo André e Jundiaí.

O objetivo do estudo foi minimizar o custo de frete para atendimento das demandas necessárias. Para encontrar a melhor solução com o menor custo de frete foi aplicado técnicas de programação linear com utilização do Solver, suplemento do Excel.

Revista ENIAC Pesquisa, Guarulhos (SP), V.7, n.1, jan.- jun. 2018. 


\section{ESTUDO DE CASO}

A empresa de varejo de materiais para construção a qual possui 2 CD's no estado de São Paulo (CDG-Guarulhos e CDB-Santo André). Os dois CD’s (Centro de Distribuição) devem se encarregar de abastecer as lojas de Francisco Morato, Anhanguera, Aricanduva, Santo André, Marginal Tietê e Jundiaí. Considerando os custos de transporte unitários, a capacidade de armazenagem e a demanda das lojas conforme tabela abaixo. Foram aplicadas técnicas de programação linear utilizando a ferramenta SOLVER do Excel para determinar quanto deve ser entregue por centro de distribuição para cada Loja consumidora, de forma a minimizar os custos de transporte. Os dados estão apresentados abaixo na Tabela 1 e o diagrama distribuído conforme FIGURA 1.

TABELA 1 - Dados de Capacidade, Demanda em unidades e Valores de Fretes

\begin{tabular}{llclclll}
\hline CD/LOJAS & $\begin{array}{l}\text { PRO- } \\
\text { MORATO } \\
(\mathbf{3})\end{array}$ & $\begin{array}{l}\text { ANHANGUERA } \\
(\mathbf{4})\end{array}$ & $\begin{array}{l}\text { PRO- } \\
\text { ARICANDUVA } \\
(\mathbf{5})\end{array}$ & $\begin{array}{l}\text { SANTO } \\
\text { ANDRÉ } \\
(\mathbf{6})\end{array}$ & $\begin{array}{l}\text { PRO- } \\
\text { MARGINAL } \\
(\mathbf{7})\end{array}$ & $\begin{array}{l}\text { JUNDIAÍ } \\
(\mathbf{8})\end{array}$ & CAPACIDADE \\
\hline CDG (1) & $2,07 \$$ & $2,07 \$$ & $2,07 \$$ & $2,07 \$$ & $2,07 \$$ & $2,63 \$$ & $\mathbf{4 0 0 0}$ \\
\hline CDB (2) & $2,63 \$$ & $2,84 \$$ & $2,63 \$$ & $2,63 \$$ & $2,63 \$$ & $2,63 \$$ & $\mathbf{3 7 0 0}$ \\
\hline $\begin{array}{l}\text { DEMAND } \\
\text { A }\end{array}$ & $\mathbf{1 0 0 0}$ & $\mathbf{2 5 0}$ & $\mathbf{1 8 0 0}$ & $\mathbf{2 5 0}$ & $\mathbf{3 3 0 0}$ & $\mathbf{4 5 0}$ \\
\hline
\end{tabular}

Fonte: Elaborado pelos autores, 2017.

Revista ENIAC Pesquisa, Guarulhos (SP), V.7, n.1, jan.- jun. 2018. 
FIGURA 1- Diagrama de rede do problema levantado.

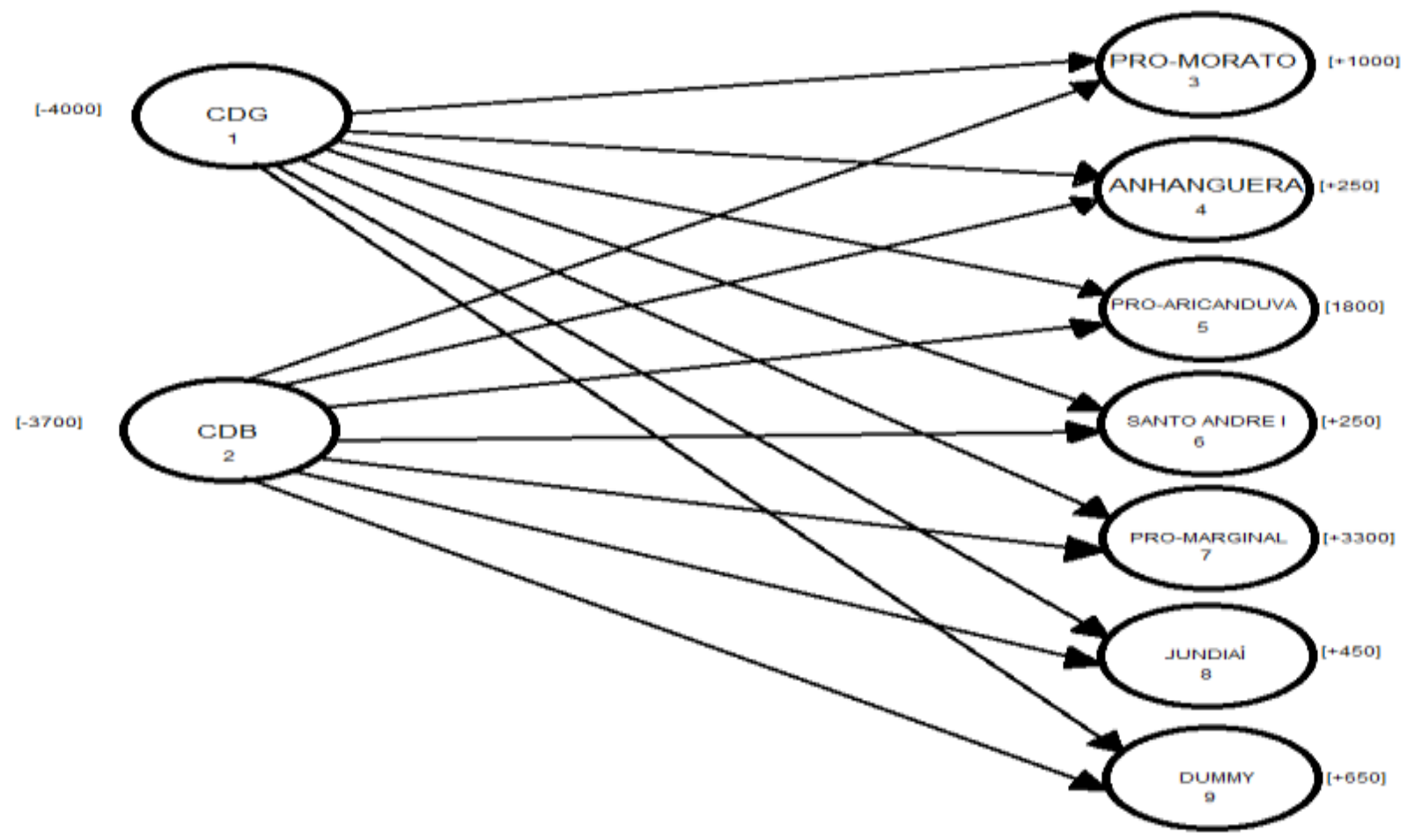

Fonte: Elaborado pelos autores, 2017.

O primeiro passo para a resolução desse problema de otimização foi a determinação das variáveis de decisão ficando relacionada da seguinte forma:

X13 - quantidade enviada do CDG para a Loja de Francisco Morato

X14 - quantidade enviada do CDG para a Loja da Anhanguera

X15 - quantidade enviada do CDG para a Loja da Aricanduva

X16 - quantidade enviada do CDG para a Loja de Santo André I

X17 - quantidade enviada do CDG para a Loja da Marginal Tietê

X18 - quantidade enviada do CDG para a Loja de Jundiaí

X19 - quantidade enviada do CDG para o Dummy

X23 - quantidade enviada do CDB para a Loja de Francisco Morato

X24 - quantidade enviada do CDB para a Loja da Anhanguera

X25 - quantidade enviada do CDB para a Loja da Aricanduva

X26 - quantidade enviada do CDB para a Loja de Santo André I

X27 - quantidade enviada do CDB para a Loja da Marginal Tietê

X28 - quantidade enviada do CDG para a Loja de Jundiaí

X29 - quantidade enviada do CDG para o Dummy

Revista ENIAC Pesquisa, Guarulhos (SP), V.7, n.1, jan.- jun. 2018. 
Após a determinação das variáveis de decisão e considerando que se trata de um problema para minimização do custo total de transporte, chegamos a seguinte função objetivo:

$$
\begin{aligned}
& \text { Minimização }=2,07 \mathrm{X} 13+2,07 \mathrm{X} 14+2,07 \mathrm{X} 15+2,07 \mathrm{X} 16+2,07 \mathrm{X} 17+ \\
& 2,63 \mathrm{X} 18+2,63 \mathrm{X} 23+2,84 \mathrm{X} 24+2,63 \mathrm{X} 25+2,63 \mathrm{X} 26+2,63 \mathrm{X} 27+2,63 \mathrm{X} 28
\end{aligned}
$$

Pare o problema em questão teremos algumas restrições tanto para os centros de distribuição quanto para as lojas consumidoras, são elas;

$$
\begin{aligned}
& \text { Nó 1) }-\mathrm{X} 13-\mathrm{X} 14-\mathrm{X} 15-\mathrm{X} 16-\mathrm{X} 17-\mathrm{X} 18-\mathrm{X} 19=-4000 \\
& \text { Nó 2) }-\mathrm{X} 23-\mathrm{X} 24-\mathrm{X} 25-\mathrm{X} 26-\mathrm{X} 27-\mathrm{X} 28-\mathrm{X} 29=-3700 \\
& \text { Nó 3) } \mathrm{X} 13+\mathrm{X} 23=1000 \\
& \text { Nó 4) } \mathrm{X} 14+\mathrm{X} 24=250 \\
& \text { Nó 5) } \mathrm{X} 15+\mathrm{X} 25=1800 \\
& \text { Nó 6) } \mathrm{X} 16+\mathrm{X} 26=250 \\
& \text { Nó 7) } \mathrm{X} 17+\mathrm{X} 27=3300 \\
& \text { Nó 8) } \mathrm{X} 18+\mathrm{X} 28=450 \\
& \text { Nó 9) } \mathrm{X} 19+\mathrm{X} 29=650 \\
& \mathrm{X}>=0 \text { Para i }=1-2 \text { e } \mathrm{j}=1-9
\end{aligned}
$$

A última restrição impede que o resultado dos cálculos apresente algum valor negativo. Após aplicação do método acima, chegamos à solução ótima conforme Figura 2 .

Revista ENIAC Pesquisa, Guarulhos (SP), V.7, n.1, jan.- jun. 2018. 
FIGURA 2 - Solução ótima utilizando métodos quantitativos.

\begin{tabular}{|r|l|r|l|r|r|}
\hline \multicolumn{2}{|c|}{ De } & \multicolumn{2}{c|}{ Para } & CUSTO & UNIDADE \\
\hline Nó & Centro & Nó & Loja & & \\
\hline 1 & CDG & 3 & PRO-MORATO & 2,07 & 1000 \\
\hline 1 & CDG & 4 & ANHANGUERA & 2,07 & 250 \\
\hline 1 & CDG & 5 & PRO-ARICANDUVA & 2,07 & 1800 \\
\hline 1 & CDG & 6 & SANTO ANDRÉ I & 2,07 & 250 \\
\hline 1 & CDG & 7 & PRO-MARGINAL & 2,07 & 700 \\
\hline 1 & CDG & 8 & JUNDIÁ & 2,63 & 0 \\
\hline 1 & CDG & 9 & DUMMY & 0 & 0 \\
\hline 2 & CDB & 3 & PRO-MORATO & 2,63 & 0 \\
\hline 2 & CDB & 4 & ANHANGUERA & 2,84 & 0 \\
\hline 2 & CDB & 5 & PRO-ARICANDUVA & 2,63 & 0 \\
\hline 2 & CDB & 6 & SANTO ANDRÉ I & 2,63 & 0 \\
\hline 2 & CDB & 7 & PRO-MARGINAL & 2,63 & 2600 \\
\hline 2 & CDB & 8 & JUNDIAÍ & 2,63 & 450 \\
\hline 2 & CDB & 9 & DUMMY & 0 & 650 \\
\hline
\end{tabular}

\begin{tabular}{|r|l|r|r|}
\hline Nó & Centro/loja & Fluxo Liquido & oferta demanda \\
\hline 1 & CDG & -4000 & -4000 \\
\hline 2 & CDB & -3700 & -3700 \\
\hline 3 & PRO-MORATO & 1000 & 1000 \\
\hline 4 & ANHANGUERA & 250 & 250 \\
\hline 5 & PRO-ARICANDUVA & 1800 & 1800 \\
\hline 6 & SANTO ANDRÉ I & 250 & 250 \\
\hline 7 & PRO-MARGINAL & 3300 & 3300 \\
\hline 8 & JUNDIÁ & 450 & 450 \\
\hline \multicolumn{2}{r|}{ 9 DUMMY } & 650 & 650 \\
\hline \multicolumn{2}{|c|}{ Oferta+demanda } & 0 \\
\cline { 2 - 4 }
\end{tabular}

Fonte: Elaborado pelos autores, 2017.

Foi aplicado o método de aproximação de Vogel, primeiramente deve-se calcular a diferença entre os menores custos de frete e colocado no campo diferença conforme Tabela 2.

TABELA 2 - Método de aproximação de Vogel $1^{\text {a }}$ Interação

\begin{tabular}{|c|c|c|c|c|c|c|c|c|}
\hline 20 & $\begin{array}{c}\text { PRO- } \\
\text { MORAT } \\
\text { O (3) }\end{array}$ & $\begin{array}{c}\text { ANHANGUERA } \\
\text { (4) }\end{array}$ & $\begin{array}{c}\text { PRO- } \\
\text { ARICANDUVA } \\
\text { (5) }\end{array}$ & $\begin{array}{c}\text { SANTO } \\
\text { ANDRÉ I } \\
(6)\end{array}$ & $\begin{array}{c}\text { PRO- } \\
\text { MARGINAL } \\
(7)\end{array}$ & $\begin{array}{c}\text { JUNDIAÍ } \\
\text { (8) }\end{array}$ & CAPACIDADE & DIF. \\
\hline CDG (1) & 2,07 & 2,07 & 2,07 & 2,07 & 2,07 & 2,63 & 4000 & 0 \\
\hline CDB (2) & 2,63 & 2,84 & 2,63 & 2,63 & 2,63 & 2,63 & 3700 & 0 \\
\hline DEMANDA & 1000 & 250 & 1800 & 250 & 3300 & 450 & & \\
\hline $\begin{array}{l}\text { DIFERENÇ } \\
\text { A }\end{array}$ & 0,56 & 0,77 & 0,56 & 0,56 & 0,56 & 0 & & \\
\hline
\end{tabular}

Fonte: Elaborado pelos autores, 2017.

Após cálculos das diferenças, deve se trabalhar em cima da maior diferença que no caso acima é 0,77 . Após identificado a maior diferença, devemos selecionar o menor custo da coluna que no caso é 2,07 e com isso atenderemos a loja Anhanguera pelo CDG com a demanda total. Esse processo deverá ser feito até atender todas as

Revista ENIAC Pesquisa, Guarulhos (SP), V.7, n.1, jan.- jun. 2018. 
demandas. Após todas as interações o resultado encontrado está apresentado na Tabela 3 e, consequentemente, os valores são conforme a Tabela 4.

TABELA 3 - Método de Aproximação de Vogel

\begin{tabular}{|c|c|c|c|c|c|c|c|}
\hline & $\begin{array}{c}\text { F. } \\
\text { MORATO }\end{array}$ & ANHANGUERA & ARICANDUVA & $\begin{array}{c}\text { Sto. } \\
\text { ANDRÉ }\end{array}$ & MARGINAL & JUNDIAÍ & OFERTA \\
\hline CD1 & $\begin{array}{r}2,07 \\
\mathbf{2 0 0}\end{array}$ & $\begin{array}{r}2,07 \\
\mathbf{2 5 0}\end{array}$ & 2,07 & $\begin{array}{r}2,07 \\
\mathbf{2 5 0}\end{array}$ & $\begin{array}{r}2,07 \\
3300\end{array}$ & 2,63 & $\begin{array}{r}4000 \\
-250 \\
-3000 \\
-250 \\
-200 \\
0\end{array}$ \\
\hline CD2 & $\begin{array}{r}2,63 \\
800\end{array}$ & 2,84 & $\begin{array}{r}2,63 \\
\mathbf{1 8 0 0}\end{array}$ & 2,63 & 2,63 & $\begin{array}{r}2,63 \\
\mathbf{4 5 0}\end{array}$ & $\begin{array}{r}3700 \\
-1800 \\
-800 \\
-450 \\
\\
650\end{array}$ \\
\hline D & 1000 & 250 & 1800 & 250 & 3300 & 450 & \\
\hline
\end{tabular}

Fonte: Elaborado pelos autores, 2017.

TABELA 4 - Solução Ótima pelo Método de Aproximação de Vogel

\begin{tabular}{llrrrr}
\hline DE & \multicolumn{1}{c}{ PARA } & CUSTO & UNIDADE & & \multicolumn{1}{c}{ VALOR } \\
\hline CD1 & ANHANGUERA & 2,07 & 250 & $\mathrm{R} \$$ & 517,50 \\
CD1 & MARGINAL & 2,07 & 3300 & $\mathrm{R} \$$ & $6.831,00$ \\
CD1 & SANTO ANDRÉ & 2,07 & 250 & $\mathrm{R} \$$ & 517,50 \\
CD1 & FRANCISCO & 2,07 & 200 & $\mathrm{R} \$$ & 414,00 \\
& MORATO & & & & \\
CD2 & FRANCISCO & 2,63 & 800 & $\mathrm{R} \$$ & $2.104,00$ \\
& MORATO & & & \\
CD2 & ARICANDUVA & 2,63 & 1800 & $\mathrm{R} \$$ & $4.734,00$ \\
CD2 & JUNDIAÍ & 2,63 & 450 & $\mathrm{R} \$$ & $1.183,50$ \\
& & TOTAL & 7050 & $\mathrm{R} \$$ & $16.301,50$ \\
\hline
\end{tabular}

Fonte: Elaborado pelos autores, 2017.

\section{CONSIDERAÇÕES FINAIS}

Essa pesquisa amplia os nossos conhecimentos acerca das técnicas de programação linear, mediante aplicação em um problema real de minimização dos custos de distribuição. A área de estudo foi selecionada em função dos impactos financeiros e operacionais das técnicas que ela dispõe, pois, a pesquisa operacional, conforme mencionado na revisão de literatura, é uma área recheada de várias técnicas de otimização de operações.

Analisando os resultados de ambas as técnicas apresentadas observa-se que em termos de resultado final no que se refere aos custos, o resultado é o mesmo, tanto a

Revista ENIAC Pesquisa, Guarulhos (SP), V.7, n.1, jan.- jun. 2018. 
programação linear por diagrama de rede como por método de Vogel, o custo final de distribuição foi de 16.301,50 reais, a única diferença resume-se em quem manda para quem, pois na programação linear com diagrama de rede por exemplo mostrou que a loja de Pro-Morato recebe as 1000 unidades de sacos de cimento apenas do Centro de Distribuição de Guarulhos, porém, no método de Vogel as 1000 unidades de ProMorato viriam dos dois centros de distribuição sendo, 200 unidades do Centro de Distribuição de Guarulhos e as demais 800 do Centro de Distribuição de Santo André.

Os resultados do presente estudo corroboram a literatura no que se refere a redução de custos na logística de distribuição por meio de técnicas de otimização linear que dispõe a pesquisa operacional uma vez que os resultados apresentados sempre atenderão o objetivo proposto, nesse caso a minimização. Uma aplicação como essa pode evitar que uma empresa tenha mais gastos do que o necessário devido a decisões empíricas por parte dos gestores responsáveis da atividade de transporte e distribuição. Como sugestão para futuras pesquisas poderia ser considerado o transporte dos sacos de cimento em lotes com quantidades determinadas pela capacidade dos veículos disponíveis.

\section{REFERÊNCIAS}

Caixeta-Filho, J. V. (2015). Gestão Logística do Transporte de Cargas. $2^{\mathrm{a}}$ ed. Atlas S.A.

Couto, Cristiane dos Santos. (2015). Uso de métodos quantitativos na determinação da equação de custo da empresa. Monografia apresentada para a obtenção do Certificado de Especialização em MBA Gerência Financeira e Controladoria do Departamento de Economia, Contabilidade e Administração da Universidade de Taubaté. disponível em : <http://www.unisc.br/portal/upload/com_arquivo/uso_de_metodos_quantitativos_na_de terminacao_da_equacao_de_custo_da_empresa.pdf> Acesso em: 16 nov. de 2015.

Fleury, P. F. (2002). Gestão estratégica do transporte. Revista Tecnologística, v. 82.

Frossard, A. C. P. (2009). Programação linear: maximização de lucro e minimização de custos. Revista Científica da Faculdade Lourenço Filho, São Paulo, v. 6, p. 19-48, 2009.

Lachtermacher, G. (2009). Pesquisa operacional na tomada de decisões, $4^{\text {a }}$ Ed., Prentice Hall, p.118.

Marins, F. A. S. (2011). Introdução à Pesquisa Operacional. São Paulo: Cultura

Revista ENIAC Pesquisa, Guarulhos (SP), V.7, n.1, jan.- jun. 2018. 
Acadêmica: Universidade Estadual Paulista.

Martín, Q. M. (2015). Investigación operativa; Madrid: Pretence hall, 2003 apud METODOS QUANTITATIVO - Cap. 1 e 2 introdução \& estatística básica, disponível em: <http://pt.slideshare.net/ElvisMagno/cap-1-e-2-introduo-estatsticas-bsica> acesso em : 15 de nov. de 2015.

Moreira, D. A. (2010). Pesquisa Operacional, Curso Introdutório, $2^{\circ}$ edição revista atualizada, São Paulo.

Novaes, A. (2007). Logística e Gerenciamento da Cadeia de Distribuição. $3^{a}$ ed. Elsevier.

Pedroso Neto, D. et al. (2015). Custos Logísticos. São Paulo: Scortecci Editora.

Pompermayer, C. B.; Lima, J. E. P. (2002). Gestão de custos. Finanças empresariais/FAE Business School. Curitiba: Associação Franciscana de Ensino Senhor Bom Jesus, v. 4, p. 49-68.

Rezende Filho, M. (2006). Programação Linear - Otimização de Recursos em Apoio à Tomada de Decisão. Rio de Janeiro: Fábrica do livro. V.1.

Simons JR. J.V (1992). Heuristics in flow shop scheduling with sequence dependente setup times. Omega - The International Journal of Management Science, Oxford, v.20, n2,p215-225.

Tadeu, Hugo Ferreira Braga. métodos quantitativos aplicado na gestão de estoques um estudo de caso prático para as organizações, disponível em: <http://www.techoje.com.br/site/techoje/categoria/detalhe_artigo/658> acesso em: 08 de out. de 2015.

Revista ENIAC Pesquisa, Guarulhos (SP), V.7, n.1, jan.- jun. 2018. 